\title{
Rectal gonorrhoea as an independent risk factor for HIV infection in a cohort of homosexual men
}

Kevin J P Craib, David R Meddings, Steffanie A Strathdee, Robert S Hogg, Julio S G Montaner, Michael V O'Shaughnessy, Martin T Schechter

\begin{abstract}
Objective-To determine whether certain sexually transmitted diseases are independent risk factors for HIV transmission in a cohort of homosexual men.

Methods-Eligible cases were identified as those who had seroconverted between November 1982 and November 1990. Two persistently HIV-seronegative control participants were randomly selected for each case from all participants who remained seronegative in November 1990. For cases, risk factor data were taken from an index visit which was defined as the first seropositive visit, while for controls these data were obtained from a matched visit which occurred within two months of the index visit for the corresponding case. MantelHaenszel methods and logistic regression were used to compare differences in risk factors for seroconversion between cases and controls.
\end{abstract}

Results-A total of 125 cases and 250 controls were eligible for this study. Cases were significantly more likely to have had reported any gonorrhoea $(17 \%$ versus $6 \%$; OR = 2.94; 95\% CI: $1 \cdot 51-5 \cdot 73)$ or syphilis ( $7 \%$ versus $2 \%$; OR $=3.78 ; 95 \%$ CI: 1.33-10.79) than controls during the seroconversion period. Multivariate logistic regression revealed rectal gonorrhoea to be independently associated with risk of seroconversion (odds ratio $=3 \cdot 18 ; p=$ $0 \cdot 044)$, whereas urethral gonorrhoea $(p=$ $0.479)$ and pharyngeal gonorrhoea $(p=$ $0.434)$ were not after inclusion of rectal gonorrhoea. In addition, the following variables were also shown to exert an independent effect on seroconversion: frequency of anal intercourse, use of illicit drugs, number of male sexual partners, and lack of a post-secondary education.

Conclusions-In this observational study, rectal gonorrhoea was found to be associated with HIV seroconversion after adjustment for a number of HIV risk factors. We cannot rule out that rectal gonorrhoea was not directly associated with HIV infection but rather with other residual lifestyle factors not fully adjusted for in the analysis. However, the relationship with gonococcal involvement of a specific anatomic site lends support to a biological association between gonorrhoea and HIV infection, rather than to alternative non-biologic explanations. Our findings are consistent with previous studies reporting an association between HIV infection and non-ulcerative sexually transmitted diseases. Such a direct association might be explained by postulating that gonorrhoea results in inflamed rectal mucosa and compromised epithelial integrity, thereby predisposing an individual to subsequent HIV infection.

(Genitourin Med 1995;71:150-154)

Keywords: HIV; seroconversion; sexually transmitted diseases; gonorrhoea; homosexual men

Introduction

There is growing evidence that sexually transmitted diseases act as important cofactors for HIV transmission. In sub-Saharan Africa, heterosexual transmission of HIV has been shown to be enhanced by genital or anorectal ulcerative disease, largely due to chancroid. ${ }^{1-4}$ The hypothesis that ulcers act as portals for HIV transmission has been supported by the isolation of HIV from exudates taken from chancroid ulcers in Nairobi. ${ }^{5}$ In North America, herpes simplex virus type 2 has been shown to be a risk factor for concurrent or subsequent HIV infection in homosexual men. ${ }^{67}$

A potential association between non-ulcerative diseases such as chlamydia infection or gonorrhoea and HIV transmission has been less well described. ${ }^{8}$ However, a recent study in Zaire found chlamydia, gonorrhoea, and trichomoniasis to all be independently associated with HIV seroconversion in women after simultaneously controlling for condom use and number of sexual partners. ${ }^{9}$ A Kenyan study has also shown genital chlamydia infection to be associated with heterosexual HIV transmission. ${ }^{3}$ In North America there has been limited evidence of an association between gonorrhoea and seroconversion in both heterosexuals and homosexuals. ${ }^{1011}$

In this study, we conducted a nested casecontrol analysis to determine whether certain ulcerative and non-ulcerative sexually transmitted diseases act as independent risk factors for HIV infection within a cohort of homosexual men.

\section{Methods}

The methods and aims of the Vancouver Lymphadenopathy-AIDS Study (VLAS) have 
Table 1 Comparison of age, annual income, and postsecondary education for cases and controls during the seroconversion period

\begin{tabular}{|c|c|c|c|}
\hline & Cases & Controls & Pvalue \\
\hline \multicolumn{4}{|c|}{ Mean age at index visit (SD) } \\
\hline & $32 \cdot 9(6 \cdot 9)$ & $35 \cdot 0(7 \cdot 8)$ & $0 \cdot 008^{\star}$ \\
\hline \multicolumn{4}{|l|}{ Annual inc } \\
\hline $\begin{array}{l}<\$ 10000 \\
>\$ 10000\end{array}$ & $\begin{array}{l}33(27 \%) \\
91(73 \%)\end{array}$ & $\begin{array}{r}47(19 \%) \\
202(81 \%)\end{array}$ & $0.086 t$ \\
\hline \multicolumn{4}{|c|}{ Completed primary university degree } \\
\hline No & $88(72 \%)$ & $134(54 \%)$ & $0.002 \dagger$ \\
\hline
\end{tabular}

*bases on $t$ test

tbased on chi square test (uncorrected)

been described in detail. ${ }^{12}{ }^{13}$ Briefly, the VLAS is an ongoing prospective study of 1,000 homosexual men who were enrolled during two recruitment periods. From November 1982 to December 1984, a total of 729 men were recruited through six general practices in central Vancouver. Follow-up visits occurred approximately every six months until September 1986 after which participants completed visits on an annual basis. During each visit, participants completed a selfadministered questionnaire which gathered demographic data and information regarding sexual practices, history of sexually transmitted diseases, and use of illicit drugs. The questionnaire elicited information for the interval since the last scheduled visit (preceding 6 months prior to September 1986; preceding 12 months thereafter). In addition, a complete physical examination was performed and blood samples were drawn for immunologic and HIV antibody testing. All study participants were counselled by their physicians to adopt safer sex guidelines including reduction in the number of sexual partners and avoidance of unprotected sexual intercourse.

Eligible "cases" were defined as those who had entered the cohort study seronegative and had subsequently seroconverted between November 1982 and November 1990. The estimated date of seroconversion for cases was defined as the midpoint between the last negative and first confirmed HIV-positive test. For convenience, this period between the last negative and first positive antibody test result will be referred to as the "seroconversion interval". For each case, two seronegative controls were randomly selected from all participants who had remained seronegative in November 1990. For cases, data regarding risk factors

Table 2 Comparison of sexual practices in cases and controls

\begin{tabular}{lccl}
\hline & Cases $(n, \%)$ & Controls $(n, \%)$ & $\begin{array}{l}\text { Odds ratio } \\
(95 \% \text { CI })\end{array}$ \\
\hline $\begin{array}{l}\text { Receptive anal intercourse } \\
\leqslant 25 \% \text { of encounters }\end{array}$ & $64(51 \%)$ & $175(71 \%)$ & $1 \cdot 00$ \\
$>25 \%$ & $61(49 \%)$ & $72(29 \%)$ & $2 \cdot 32$ \\
& & & $(1 \cdot 49,3 \cdot 60)$ \\
Insertive anal intercourse & $50(40 \%)$ & $156(63 \%)$ & $1 \cdot 00$ \\
$\begin{array}{l}\leqslant 25 \% \text { of encounters } \\
>25 \%\end{array}$ & $75(60 \%)$ & $91(37 \%)$ & $2 \cdot 57$ \\
$\begin{array}{l}\text { Number of male sexual partners } \\
\text { (in previous year) }\end{array}$ & & $(1 \cdot 65,3 \cdot 98)$ \\
$\leqslant 5$ & $67(54 \%)$ & $165(66 \%)$ & $1 \cdot 00$ \\
$>5$ & $58(46 \%)$ & $84(34 \%)$ & $1 \cdot 70$ \\
& & & $(1 \cdot 10,2 \cdot 63)$ \\
\hline
\end{tabular}

were obtained from the first visit at which the participant was found to be seropositive, denoted the index visit. This choice was made in order to utilise the questionnaire which elicited information pertaining most closely to the seroconversion interval. For controls, risk factor data were obtained from the corresponding visit which occurred within two months of the index visit for the corresponding case.

Univariate comparisons between cases and control groups were carried out using MantelHaenszel methods. Fisher's exact test was employed when more than $25 \%$ of the expected cell frequencies were less than five. Unadjusted relative risk estimates were computed using the odds ratio. Ninety five per cent confidence intervals were approximated using test-based limits proposed by Miettinen. ${ }^{14}$ Multivariate comparisons were carried out using logistic regression analysis. For all comparisons, participants with missing or unknown values for the variable(s) of interest were excluded from the analysis. All reported p-values are two-sided.

\section{Results}

A total of 125 men seroconverted between November 1982 and November 1990 and formed the case group. Accordingly, 250 controls were selected for the comparative analysis. The median duration between the estimated date of seroconversion and the index visit for cases was 6 months (interquartile range of 3 to 8 months).

Table 1 compares cases and controls with respect to age at the index visit, and annual income and post-secondary education at enrolment. Men who seroconverted were younger than controls (mean age of 32.9 versus 35.0 years; $p=0.008$ ), less likely to have completed a university degree $(28 \%$ versus $46 \% ; p=0.001$ ) and more likely to have earned below $\$ 10,000$ per year (27\% versus $19 \% ; p=0.086)$.

We compared cases and controls with respect to the frequency of anal intercourse and number of male sexual partners during the seroconversion interval (table 2). Significantly more cases reported having practised receptive anal intercourse in more than $25 \%$ of sexual encounters ( $49 \%$ versus $29 \%$; $p$ $<0.001)$ yielding an odds ratio of 2.32 (95\% CI: 1.49 , 3.60). Similarly, $60 \%$ of cases reported insertive anal intercourse in more than $25 \%$ of encounters compared to $37 \%$ of controls $(p<0.001)$ giving rise to an odds ratio of 2.57 (95\% CI: $1.65,3.98)$. Significantly more cases reported having had five or more sexual partners during the seroconversion interval ( $46 \%$ versus $33 \% ; p=$ $0.017)$ yielding an odds ratio of $1.70(95 \%$ CI: $1 \cdot 10,2 \cdot 63)$. No significant differences between these two groups were observed with respect to condom use during anal intercourse $(p=0.250)$, the practice of receptive fisting (Fisher's $p=0 \cdot 138$ ), insertive oral intercourse $(p=0.249)$ or receptive oral intercourse $(p=$ $0 \cdot 129)$. 
Table 3 History of infectious diseases in cases and controls during the seroconversion interval

\begin{tabular}{lccl}
\hline & Cases $(n, \%)$ & Controls $(n, \%)$ & $\begin{array}{l}\text { Odds ratio } \\
(95 \% C I)\end{array}$ \\
\hline $\begin{array}{l}\text { Syphilis } \\
\text { No }\end{array}$ & $116(93)$ & $244(98)$ & $1 \cdot 00$ \\
Yes & $9(7)$ & $5(2)$ & $3 \cdot 78$ \\
& & & $(1 \cdot 33,10 \cdot 79)$ \\
Pubic lice & $89(71)$ & $199(79)$ & $1 \cdot 00$ \\
No & $36(29)$ & $50(21)$ & $1 \cdot 61$ \\
Yes & & & $(0 \cdot 98,2 \cdot 64)$ \\
Intestinal parasites & $111(89)$ & $235(94)$ & $1 \cdot 00$ \\
No & $14(11)$ & $14(6)$ & $2 \cdot 12$ \\
Yes & & & $(0 \cdot 99,4 \cdot 53)$ \\
Herpes & $96(77)$ & $202(81)$ & $1 \cdot 00$ \\
No & $29(23)$ & $47(19)$ & $1 \cdot 30$ \\
Yes & & & $(0 \cdot 77,2 \cdot 19)$ \\
Venereal warts & $107(86)$ & $215(86)$ & $1 \cdot 00$ \\
No & $18(14)$ & $34(14)$ & $1 \cdot 15$ \\
Yes & & & $(0 \cdot 57,1 \cdot 97)$ \\
Non specific urethritis & $105(84)$ & $222(90)$ & $1 \cdot 00$ \\
No & $20(16)$ & $26(10)$ & $1 \cdot 62$ \\
Yes & & & $(0 \cdot 87,3 \cdot 04)$ \\
Mononucleosis & $123(98)$ & $241(97)$ & $1 \cdot 00$ \\
No & $2(2)$ & $8(3)$ & $0 \cdot 49$ \\
Yes & & & $(0 \cdot 11,2 \cdot 28)$ \\
Hepatitis & $19(85)$ & $220(88)$ & $1 \cdot 00$ \\
No & & $29(12)$ & $1 \cdot 36$ \\
Yes & & & $(0 \cdot 73,2 \cdot 53)$ \\
& & &
\end{tabular}

Table 4 Comparison of the prior history of gonorrhoea for cases and controls during the seroconversion interval

\begin{tabular}{lccl}
\hline & Cases $(n, \%)$ & Controls $(n, \%)$ & $\begin{array}{l}\text { Odds ratio } \\
(95 \% C I)\end{array}$ \\
\hline Any gonorrhoea & $104(83)$ & $233(94)$ & $1 \cdot 00$ \\
No & $21(17)$ & $16(6)$ & $\begin{array}{l}2 \cdot 94 \\
(1 \cdot 51,5 \cdot 73)\end{array}$ \\
Yes & & & $1 \cdot 00$ \\
Pharyngeal gonorrhoea & $120(96)$ & $248(99)$ & $5 \cdot 17$ \\
No & $5(4)$ & $2(1)$ & $(1 \cdot 16,23 \cdot 00)$ \\
Yes & & & $1 \cdot 00$ \\
Rectal gonorrhoea & $114(91)$ & $245(98)$ & $4 \cdot 73$ \\
No & $11(9)$ & $5(2)$ & $(1 \cdot 75,12 \cdot 76)$ \\
Yes & & & $1 \cdot 00$ \\
Urethral gonorrhoea & $110(88)$ & $238(95)$ & $2 \cdot 75$ \\
No & $15(12)$ & $12(5)$ & $(1 \cdot 25,5 \cdot 83)$ \\
Yes & & & \\
\end{tabular}

Table 5 Final logistic regression model for factors associated with seroconversion including gonorrhoea by anatomical site

\begin{tabular}{lllll}
\hline Variable & Beta & S.E. & Odds ratio & p value \\
\hline Rectal gonorrhoea & 1.155 & 0.573 & 3.18 & 0.044 \\
Anal intercourset & 0.930 & 0.253 & 2.53 & $<0.001$ \\
Illicit drugsł & 0.611 & 0.248 & 1.84 & 0.014 \\
Number of partners $\$ & 0.499 & 0.240 & 2.00 & 0.038 \\
Education|| & 0.734 & 0.251 & 2.08 & 0.004
\end{tabular}

*self-reported rectal gonorrhoea during the seroconversion interval

treceptive or insertive anal intercourse with $\geqslant 25 \%$ of sexual partners

¥Self-reported use of amphetamine, cocaine, marijuana, MDA or poppers during seroconversion period

Smore than 5 male sexual partners in the prior interval

$\|$ did not complete university degree

Table 3 compares cases and controls with respect to infectious diseases reported since the previous questionnaire. Men who had seroconverted were more likely to have reported having had syphilis ( $7 \%$ versus $2 \%$; Fisher's $p=0 \cdot 019)$. Marginal differences were observed for intestinal parasites $(11 \%$ versus $6 \% ; p=0.053)$ and pubic lice $(29 \%$ versus $21 \% ; p=0.059)$ and no differences were detected for non-specific urethritis, herpes simplex, mononucleosis, hepatitis, and venereal warts.

Table 4 compares cases and controls with respect to their history of gonorrhoea and its localization. Significantly more cases reported a history of gonorrhoea during the seroconversion interval $(17 \%$ versus $6 \% ; p=0.002)$ yielding an odds ratio of 2.94 (95\% CI: 1.51 , $5 \cdot 73)$. In comparison with controls, men who had seroconverted were more likely to have reported having had urethral $(12 \%$ versus $5 \%$; $p=0.011$ ), rectal (9\% versus $2 \% ; p=0.002$ ), and pharyngeal gonorrhea (4\% versus $1 \%$; Fisher's $p=0.044$ ).

Table 5 presents the final multivariate logistic model for seroconversion. All variables found to be significantly or marginally associated with seroconversion in the univariate analyses were considered for inclusion in this model. Owing to the high degree of collinearity between anal insertive and anal receptive intercourse, these were modelled as a single variable representing anal intercourse, receptive or insertive. In order to determine whether seroconversion might be affected by anatomical location of gonococcal infection, the multivariate analysis was conducted with variables specifying whether gonorrhoea involved the rectum, urethra, or pharynx. Rectal gonorrhoea was independently associated with risk of seroconversion in this model (odds ratio $=3.18 ; p=0.044$ ), whereas urethral gonorrhoea $(p=0.479)$ and pharyngeal gonorrhoea $(p=0.434)$ were not after inclusion of rectal gonorrhoea. In addition, the following variables were also shown to exert an independent effect on seroconversion: frequency of anal intercourse, use of illicit drugs, number of male sexual partners, and lack of a post-secondary education.

Because some cases had long seroconversion intervals, we conducted a restricted analysis using cases whose estimated dates of seroconversion occurred within 12 months of the index visit. A total of 112 cases (and 224 controls) were eligible for this sub-analysis. The results of this multivariate regression again demonstrated anal intercourse $(p<$ $0.001)$, illicit drug use $(p=0.019)$, number of male sexual partners $(p=0.035)$, and lack of a post-secondary education $(p=0.006)$ to be independently associated with seroconversion. The regression coefficient for rectal gonorrhoea was similar to those obtained in the previous unrestricted analyses (odds ratio $=$ $2 \cdot 73$ ) although it failed to achieve statistical significance $(p=0.071)$ in this model.

\section{Discussion}

In this nested case-control study, a history of rectal gonorrhoea during the interval encompassing seroconversion was the only selfreported sexually transmitted disease that appeared to act as an independent risk factor for HIV transmission based on multivariate analyses. Rectal gonorrhoea together with frequency of anal intercourse, number of male sexual partners, use of illicit drugs, and lack of 
a post-secondary education were shown to be independently associated with HIV infection.

Our results once again confirm other epidemiological studies which have demonstrated that anal intercourse, number of sexual partners, illicit drug use, and socioeconomic status are independent risk factors for HIV transmission. The frequency of anal intercourse has been uniformly shown to be one of the more important independent behavioural risk factors for this disease. ${ }^{1516}$ Number of sexual partners has similarly been demonstrated to be an important predictor of seroconversion. ${ }^{1517}$ Heavy illicit drug use by men who had seroconverted, especially the use of nitrite inhalants, is also important considering that a number of studies ${ }^{1819}$ have shown drug use to be associated with high risk sexual behaviour among homosexual men. Finally, low socioeconomic status (as measured by variables such as education, income, and occupation) has been shown on numerous occasions to be an independent predictor of high risk taking behaviour ${ }^{20}$ and initial HIV infection and prognosis. ${ }^{21}$

There are a number of caveats regarding these data which should be discussed. The timing of our questionnaires as well as the variable window period between exposure and seroconversion do not allow us to conclude that a self-reported sexually transmitted disease such as gonorrhoea was necessarily active precisely at the time of the critical HIV exposure. This would require a longitudinal design of a large number of subjects including very frequent testing for both sexually transmitted diseases and HIV. In addition, although individual participants provided us with estimates of their number of sexual partners, we could not determine nor take into account the number of HIV-infected sexual partners to which an individual was exposed, the extent of exposure to each partner (duration and type of exposure) and the degree of infectivity of each partner. ${ }^{22}$ Finally, there may be recall problems associated with the reporting of past sexual behaviour or sexually transmitted diseases. ${ }^{23}$ Although the effect of these recall problems is difficult to estimate, several studies have shown that interview-administered and self-administered questionnaires can provide reasonably reliable data concerning sexual behaviour in homosexual men when the recall period is relatively short. ${ }^{24}{ }^{25}$ In our study, information regarding the sexual practices of the cases was obtained from the questionnaire at the first seropositive visit. Except for those men who had a seroconversion illness and suspected themselves to be positive on that basis, the cases in this study were unaware of their seropositive status at the time of the index questionnaire completion. This might have mitigated against significant recall bias.

The difficulties in analysing the complex relationship of sexual behaviours and sexually transmitted diseases are well known. ${ }^{22}$ The most important limitation in assessing a sexually transmitted disease associated with HIV transmission in an observational study concerns the inherent difficulty in distinguishing biological cofactors from non-biological mechanisms, including the inadequate control of confounders or differential misclassification within the variables under consideration for inclusion in the statistical model. Although rectal gonorrhoea remained associated with HIV seroconversion after adjustment for frequency of anal intercourse, use of illicit drugs, number of male sexual partners, and lack of a post-secondary education, the possibility must be acknowledged that rectal gonorrhoea was not directly associated with HIV infection but rather with other residual lifestyle factors not fully captured in the model. However, our finding that the observed association between rectal gonorrhoea and HIV seroconversion was both independent of a number of potential confounders, and related to gonococcal involvement of a specific anatomic site lends support to a biological association between gonorrhoea and HIV infection, rather than to the alternative non-biological explanations. Although the possibility of residual confounding exists even in a multivariate analysis, the latter explanation would not appear to account for a differential association related to site of infection as easily as an explanation invoking a direct biological mechanism facilitating transmission.

Such a direct association might be explained by postulating that gonorrhoea results in inflamed rectal mucosa and compromised epithelial integrity, thereby predisposing an individual to subsequent HIV infection. It has been hypothesised that nonulcerative diseases may facilitate HIV transmission by causing inflammation and micro-ulceration, and there have been a number of observations providing support for the biological plausibility of such an association. Genital tract inflammation has been shown to increase numbers of T-lymphocytes or macrophages in reproductive fluids of men and women, thereby increasing the pool of infected cells capable of transmitting the infection, or the number of target cells in a seronegative individual. ${ }^{26-29}$ Gonococcal urethritis has also been demonstrated to be significantly correlated with isolation of HIV proviral DNA among seropositive men presenting with urethral discharge. ${ }^{30}$

Our findings support previous studies in North America that appear to show gonorrhoea as a risk factor for HIV transmission in the spouses of seropositive participants and in homosexual men. ${ }^{10} 11$ Our findings also support a recent prospective study in Zaire demonstrating an association of gonorrhoea and seroconversion that was independent of sexual behaviours. ${ }^{9}$ Our findings apparently contrast with the findings of a study in subSaharan Africa, where gonococcal infections were not found to be related to heterosexual HIV transmission. ${ }^{3}$ This lack of association may in part be due to the fact that ulcerative diseases are endemic in this area and therefore may be masking any underlying associations between non-ulcerative diseases and HIV transmission.

The fact that rectal gonorrhoea did not 
achieve statistical significance in the subanalysis restricted to those seroconverting within 12 months of their index visit may be related to the statistical power, coupled with the fact that the association in the unrestricted analysis was only marginally significant at the 0.05 level. This supposition is supported by the fact that the coefficients (and odds ratios) for all terms in this sub-analysis were similar to the multivariate model in the primary analysis.

Within the context of an observational study it is exceptionally difficult to prove conclusively that a particular sexually transmitted disease facilitates HIV transmission. However, this nested case-control study has demonstrated an independent association between seroconversion and rectal gonorrhoea during the seroconversion interval in a cohort of homosexual men. This result lends itself more readily to explanations invoking a role for gonorrhoea as a risk factor for the transmission of HIV than alternate explanations, and underlines the importance of the need for health intervention programmes which are designed to control gonorrhoea, since such initiatives might also potentially impact rates of HIV transmission. Those who are socially and economically disadvantaged are especially at risk because for these groups rates of gonorrhoea have remained high.

This work was supported by the National Health Research Development Programme of the Department of National Health and Welfare of Canada through operating grants through a National Health Research Scholar award to D Montaner and a National Health Scientist Award to D Schechter. The authors are indebted to colleagues in the AIDS Care Group at St Paul's Hospital and to Bonnie Devlin Myrna Reginaldo, Joeane Zadra and Elizabeth Ferris for their research assistance.

1 Nsubuga $P$, Mugerwa R, Nsibambi J, et al. The association of genital ulcer disease and HIV infection at a dermatology-STD clinic in Uganda. 7 AIDS 1990;3:1002-5.

2 Latif AS, Katzenstein DA, Bassett MT, et al. Genital ulcers and transmission of HIV among couples in Zimbabwe. AIDS 1989;3:519-23.

3 Plummer FA, Simonsen JN, Cameron DW, et al. Cofactors in male-female sexual transmission of human immunodeficiency virus type 1. F Infect Dis 1991;163: 233-9.

4 Simonsen JN, Cameron DW, Gakinya MN, et al. Human mmunodeficiency virus infection among men with sexually transmitted diseases. $N$ Engl $\mathcal{Y}$ Med 1988;319:274-8.

5 Kreiss JK, Coombs R, Plummer F, et al. Isolation of human immunodeficiency virus from genital ulcers in Nairobi prostitutes. F Infect Dis 1989;160:380-4

6 Stamm WE, Handfield HH, Rompalo AM, Ashley RL, Roberts PL, Corey L. The association between genital ulcer disease and acquisition of HIV infection in homosexual men. $f A M A$ 1988;260:1429-33.

7 Holmberg SD, Stewart JA, Gerber AR, et al. Prior herpes simplex virus type 2 infection as a risk factor for HIV infection. $¥ A M A$ 1988;259:1048-50.
8 Aral SO, Holmes KK. Epidemiology of sexual behavior and sexually transmitted diseases. In: Holmes $\mathrm{KK}$ Mårdh PA, Sparling PF, Wiesner PJ, eds. Sexuall Transmitted Diseases, 2nd ed New York: McGraw-Hill Information Services Company, 1990:19-36.

9 Laga M, Abib M, Mayimona K, et al. Non-ulcerative sexually transmitted diseases as risk factors for HIV-1 transmission in women: results from a cohort study. AIDS 1993;7:95-102.

10 Haley CE, Anderson P, Freeman A, Petty A. Relationship of STD history to HIV seropositivity in a cohort of homosexual men in Dallas, Texas. Int Conf AIDS 1989 June 4-9;5:126 (abstract no. W.A.P.39).

11 Fischl M, Fayne T, Flanagan S, et al. Seroprevalence and risks of HIV infections in spouses of persons infected with HIV. Int Conf AIDS 1988 June 12-16; 1:(abstract no. 4060).

12 Schechter MT, Boyko WJ, Douglas B, et al. The Vancouver Lymphadenopathy-AIDS Study, 6: HIV seroconversion in a cohort of homosexual men. Can Med Assoc F 1986;135:1355-60.

13 Schechter MT, Craib KJP, Le TN, Willoughby B, et al. Progression to AIDS and predictors of AIDS in seroprevalent and seroincident cohorts of homosexual men. prevalent and seroincider

14 Miettinen $O$. Estimability and estimation in case-referent studies. Am $\mathcal{f}$ Epidemiol 1976;103:226-35.

15 Darrow WW, Echenberg DF, Jaffe HW, et al. Risk factors for human immunodeficiency virus (HIV) infections in homosexual men. Am $\Im$ Publ Health 1987;77:479-83.

16 Kingsley LA, Detre K, Kelsey S, et al. Risk factors for seroconversion to human immunodeficiency virus among male homosexuals. Lancet 1987;1:345-9.

17 Winkelstein W Jr, Lyman DM, Padian N, et al. Sexual practices and risk of infection by the human immunodeficiency virus. The San Francisco Men's Health Study. ҒAMA 1987;257:321-5.

18 Stall R, McKusick L, Wiley J, Coates TJ, Ostrow DG. Alcohol and drug use during sexual activity and compliAlcohol and drug use during sexual activity and compli-
ance with safe sex guidelines for AIDS: The AIDS ance with safe sex guidelines for AIDS: The AIDS Behavioral
359-71.

19 Ostrow DG, VanRanden MJ, Fox R, et al. Recreational drug use and sexual behavior change in a cohort of homosexual men. AIDS 1990;4:759-65.

20 Hogg RS, Craib K, Willoughby B, Sestak P, Montane JSG, Schechter MT. Sociodemographic correlates for risk taking behavior among seronegative men. Canadian fournal of Public Health 1993;84:423-6.

21 Hogg RS, Strathdee SA, Craib KJP, O'Shaughnessy MV Montaner JSG, Schechter MT. Lower socioeconomic status and shorter survival following HIV infection. Lancet 1994;344:1120-4.

22 Mertens TE, Hayes RJ, Smith PJ. Epidemiological methods to study the interaction between HIV infection and ods to study the interaction between HIV infection and

23 Saltzman SP, Stoddard AM, McCusker J, et al. Reliability of self-reported behavior risk factors for HIV infection in homosexual men. Public Health Rep 1987;102:692-7.

24 Spanier GB. Use of recall data in survey research on human sexual behavior. Soc Biol 1976;23:244-53.

25 Coates RA, Soskolne CL, Calzavara L, et al. The reliability of sexual histories in AIDS-related research: Evaluation of an interview-administered questionnaire. Can $\mathcal{F} \mathrm{Pub}$ Health 1986;77:343-8.

26 Van de Perre P, De Clerq A, Cognaiux-Leclerc J, Nzaramba D, Butzler J-P, Sprecher-Goldberger S. Detection of HIV p17 antigen in lymphocytes but not epithelial cells from cervicovaginal secretions of women seropositive for HIV: implications for heterosexual transseropositive for Hiv: implications for heterosexual tran
mission of the virus. Genitourin Med 1988;64:30-3.

27 Wolff H, Anderson DJ. Male genital tract inflammation associated with increased numbers of potential human immunodeficiency virus host cells in semen. Andrologia immunodeficiency

28 Alexander N. Sexual transmission of human immunodeficiency virus: virus entry into male and female genital tract. Fertil Steril 1990;54:1-18.

29 Kreiss J, Carael M, Meheus A. Role of sexually transmitted diseases in transmitting human immunodeficiency virus. Genitourin Med 1988;64:1-2.

30 Moss GB, Hensel M, Emerman M, et al. Prevalence and correlates of HIV in urethral secretions of men with purulent urethritis. Int Conf AIDS 1992 July 19-24; 8(2):C327 (abstract no. PoC 4497). 